\title{
A new cone-friction test for evaluating friction phenomena in extrusion processes
}

\author{
C. Karadogan ${ }^{1, a}$, R. Grueebler ${ }^{1, b}$ and P. Hora ${ }^{1, c}$
}

${ }^{1}$ ETH Zurich, Institute of virtual manufacturing, Tannenstr. 3, 8092 Zurich, Switzerland

akaradogan@ivp.mavt.ethz.ch, ${ }^{b}$ grueebler@ivp.mavt.ethz.ch, ${ }^{c}$ hora@ivp.mavt.ethz.ch

\section{Keywords: Friction modeling, Aluminum extrusion}

\begin{abstract}
Friction is one of the most influential parameters in extrusion processes. The flow distribution is controlled using the effect of friction lengths on the extrusion dies. A comparison between multi-hole die aluminum extrusion experiments and simulations [1] necessitated studies on numerical and physical modeling of friction during aluminum extrusion. In order to measure the friction under conditions similar to extrusion processes a new experimental setup is proposed.
\end{abstract}

\section{Introduction and Motivation}

Extrusion process is highly sensitive to frictional effects. This was shown by the multi-hole die extrusion experiments of the IVP benchmark 2005 [1,2]. The die (Fig. 1 left) had 5 cylindrical outlets: One of them being central, the others lie on the same central circle, where the only difference among the holes is their diameters. The different profile lengths demonstrate that even small deviations in the shape of channels have significant influence on the velocity distribution.

The presented FEM simulations at that time mostly failed to predict this sensitive behavior correctly (Fig. 1 right). The reason was not only the lack of correct friction coefficients, which led to the use of constant values for friction together with conventional friction modeling like the Coulomb, shear or shear-limited-Coulomb, but a too simplified FEM implementation of the friction models as well. These results triggered a study on the development of new friction model implementations for extrusion process simulations, which are published in [3]. The recent goal is to specify the friction coefficient as a function of temperature, pressure and relative velocity. This study aims to develop an experimental setup to investigate such relations.
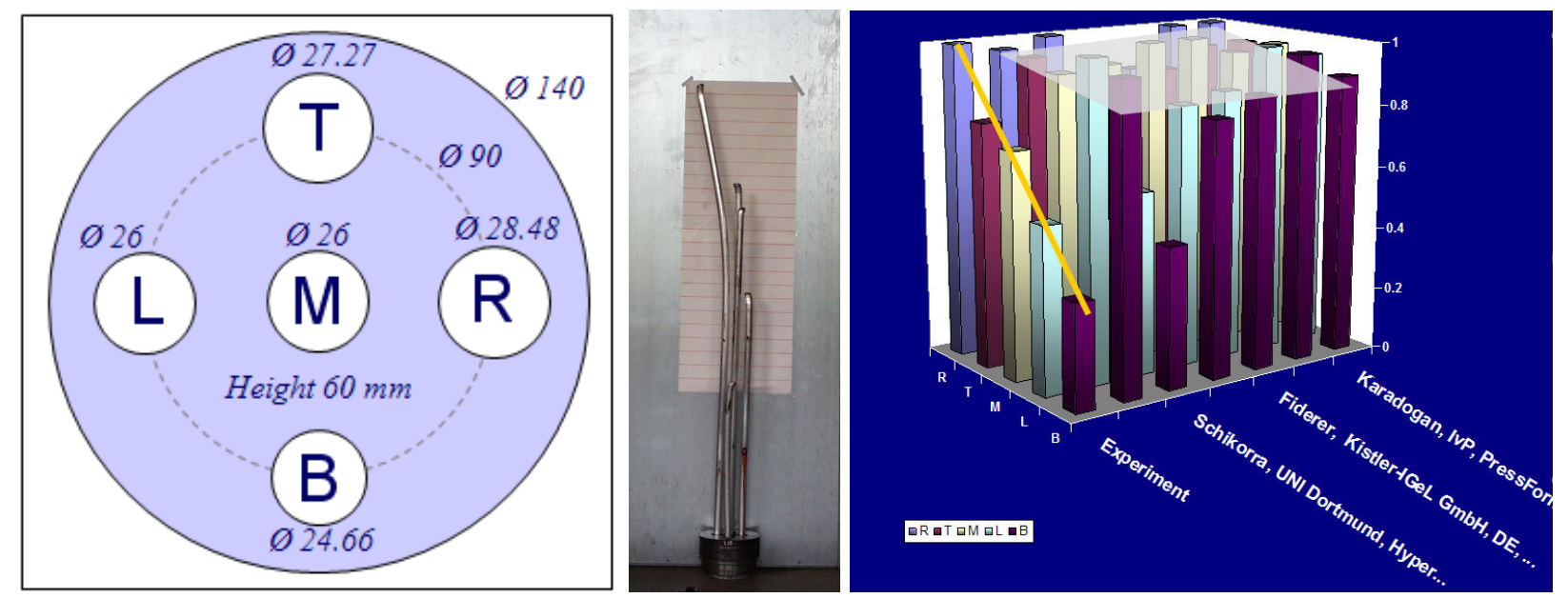

Fig. 1: Benchmark Extrusion Zurich 2005. Cooperators: SPZ- TU-Berlin \& WEFA Test Die 1.15. 


\section{Friction behavior and mathematical models}

Most of the finite element codes make use of the classical Coulomb friction model with $\tau=\mu p_{n}$, or shear friction model with $\tau=m \sigma_{Y}$. In both cases, the friction parameters $\mu$ and $m$ are assumed to be constant. The Coulomb friction model has the incorrect limits of $\tau=0$ for $p_{n}=0$, and $\tau=\infty$ for $p_{n}=\infty$. Shear friction model has again the lower limit of $\tau=0$ for the vanishing strain rate, $\dot{\gamma}=0$, which is not correct either.

The observed contamination on the aluminum extrusion dies are all clues for the sticking of the material. Additionally the friction has to be described as a function of pressure, temperature and effective relative velocity

$$
\tau=\tau\left(p, T, v_{r e l}\right)
$$

Fig. 2 shows the boundary region of the profile, where complex friction and thermal conditions are active. Recent studies [4,5] have confirmed the dependence of the friction coefficients on the process parameters pressure, temperature and velocity.

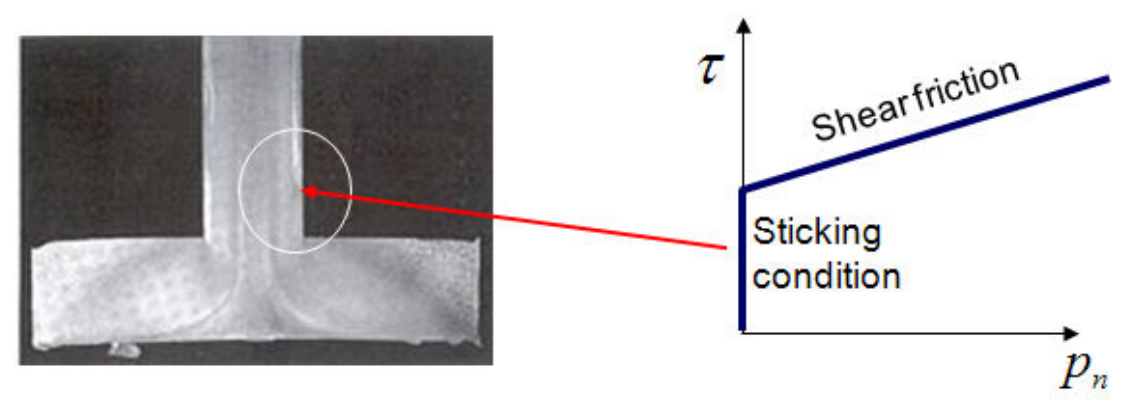

Fig. 2: Assumed frictional behavior during hot aluminum extrusion.

\section{Experimental methods}

In the above mentioned aspects, neither the pin-on-disk setup, Fig. 3, nor the ring upsetting technique [6] provides physically dependable values for the friction modeling used in extrusion process simulations. High pressure also produces side effects in the pin-on-disk investigation. Furthermore, the nature of contact attained in the pin-on-disk setup is not similar to that of extrusion. The achieved relative velocities in a ring upsetting test are much lower than the values attained in extrusion. Furthermore, the pressure, for which the corresponding friction coefficient is measured, is averaged to a greater extent in a ring upsetting test.

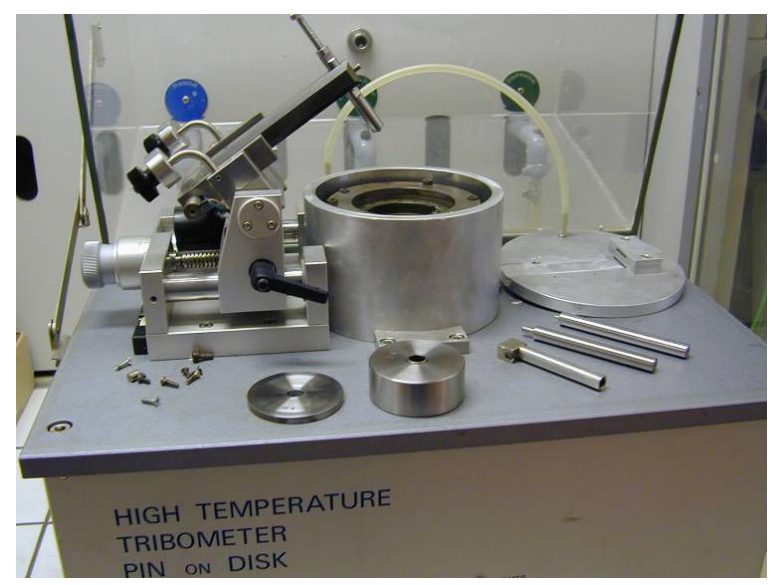

Fig. 3: High temperature pin-on-disk tribometer. 


\section{Cone-friction test}

To investigate the friction behavior in the extrusion process a new friction test setup has been developed. The setup uses a torsion testing machine as the testing facility, Fig. 4 left, and consists of a conical inner-tool in a conical aluminum ring (specimen) lying in an exterior-tool having a conical shape, Fig. 4 right. A schematic layout of the tool assembly is shown in Fig. 5. This assembly is then axially and rotationally loaded on the torsion testing machine, so that the ring plastifies under these loading conditions. The axial loading on the equipment is kept constant for each test. This is realized by first pressing the assembly axially up to a specified axial force. Then, keeping this axial force constant during the whole test, relative rotation is given to the assembly with a specified velocity. The required torque is then measured during the whole test.

The system could be heated and kept at a specific temperature and the torque could be measured for different pressures and (rotational) velocities, allowing the friction to be expressed as a function of pressure, temperature and velocity. Fig. 6 shows the finite element computation of the pressure distribution, before the beginning of the rotational loading, on the inner-tool interface for a relatively long specimen. This distribution will tend to a uniform distribution after some rotations. Even for the shown pressure distribution, the pressure fluctuation is less than $10 \%$ for the majority of the contact surface.

The experimental observations show that the inner contact interface tends to slip early. Therefore all the analytical computation of the friction is based on the inner contact interface. After an amount of relative rotation the axial force balance is due only to normal stresses on the conical surface. This leads to the following relation

$$
F_{N}=\frac{F_{\text {axial }}}{\sin \alpha}
$$

where $F_{N}$ is the total normal force on the contact interface, $F_{\text {axial }}$ is the axial force loaded by the plastometer, and $\alpha$ is the angle of conicity. Furthermore, the moment balance is expressed by

$$
M=\mu F_{N} R^{a v e}
$$

where the torque $M$ measured by the torsion testing machine is a function of normal force, friction coefficient, $\mu$, and the average radius, $R^{\text {ave }}$ of the inner cone. Combining (2) and (3) in one equation
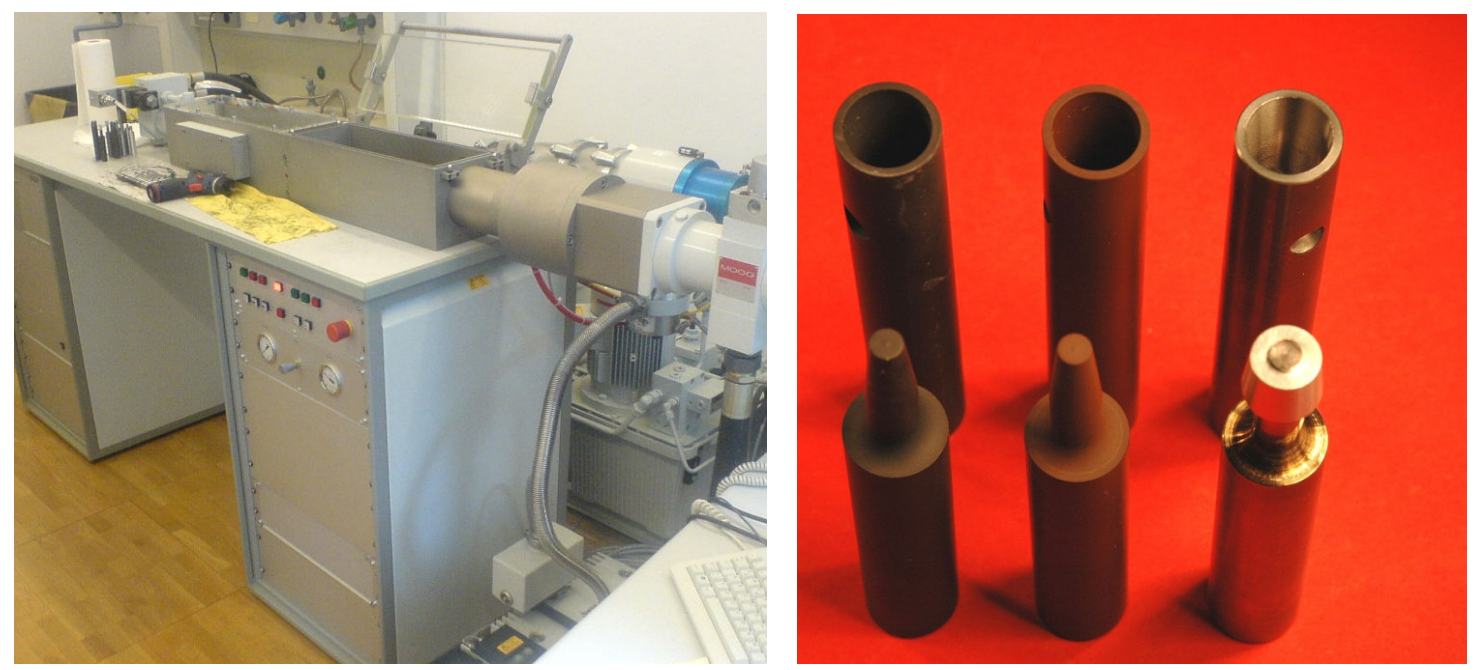

Fig. 4: Torsion testing machine, three tool-couples with different coatings and the specimen. 


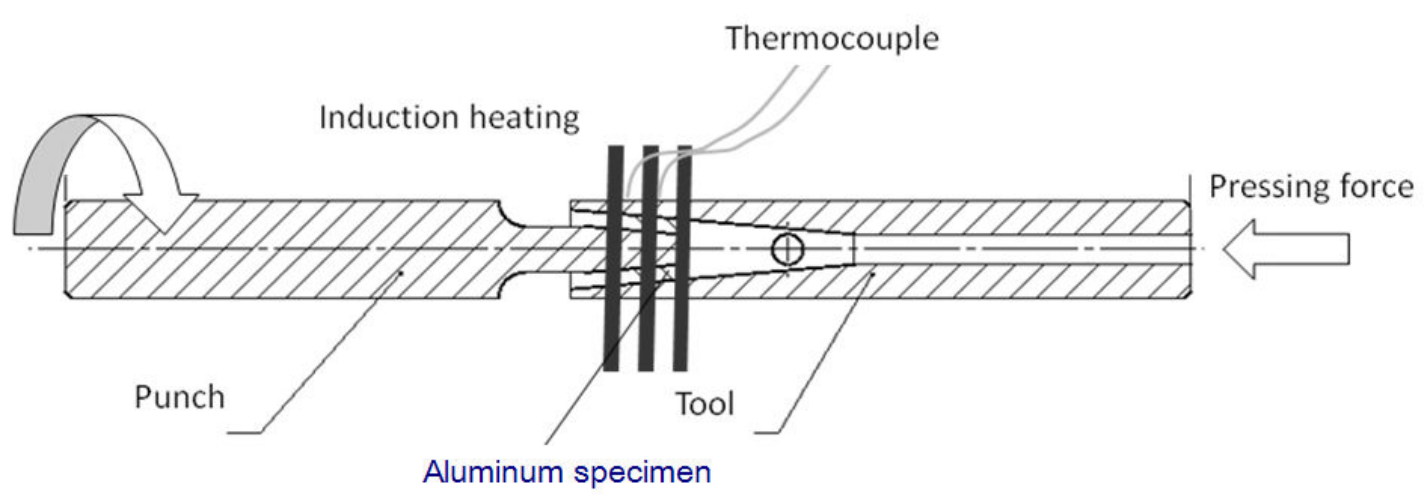

Fig. 5: Schematic layout of the friction test.

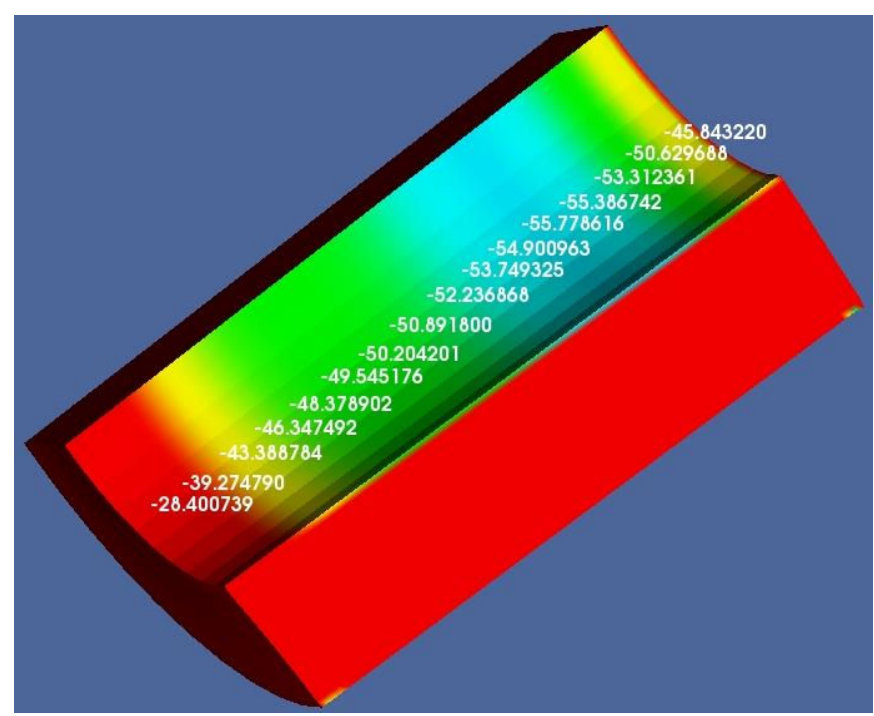

Fig. 6: Pressure distribution on the inner contact interface.

$$
\mu=\frac{M \sin \alpha}{F^{\text {axial }} R^{\text {ave }}}
$$

gives the friction coefficient. This value of the coefficient is valid for the specified temperature, velocity and normal pressure at inner contact interface. An average value for the normal pressure could be computed either analytically using $F_{N}$, or using a finite element computation. Since global balance equations are in either case fulfilled, both give the same result.

\section{Experimental results}

Selecting different materials (for the tools and specimen) and coatings, this test setup makes it possible to measure different influences on the friction. For the current study, the influence of coatings was investigated. Experiments for an uncoated tool and two different coatings have been performed. In Figures $7 \& 8$ the resulting torque is shown for two different axial pressing forces. The fluctuations in the torque measurements are due to the small misalignment of the inner and exterior tools. The uncoated tool results in the highest torque whereas the coatings 1 and 2 results in lower torques depending of the pressing forces. Details of the materials and the coatings are given in [7], another publication of this conference by our industrial collaboration partner WEFA. 


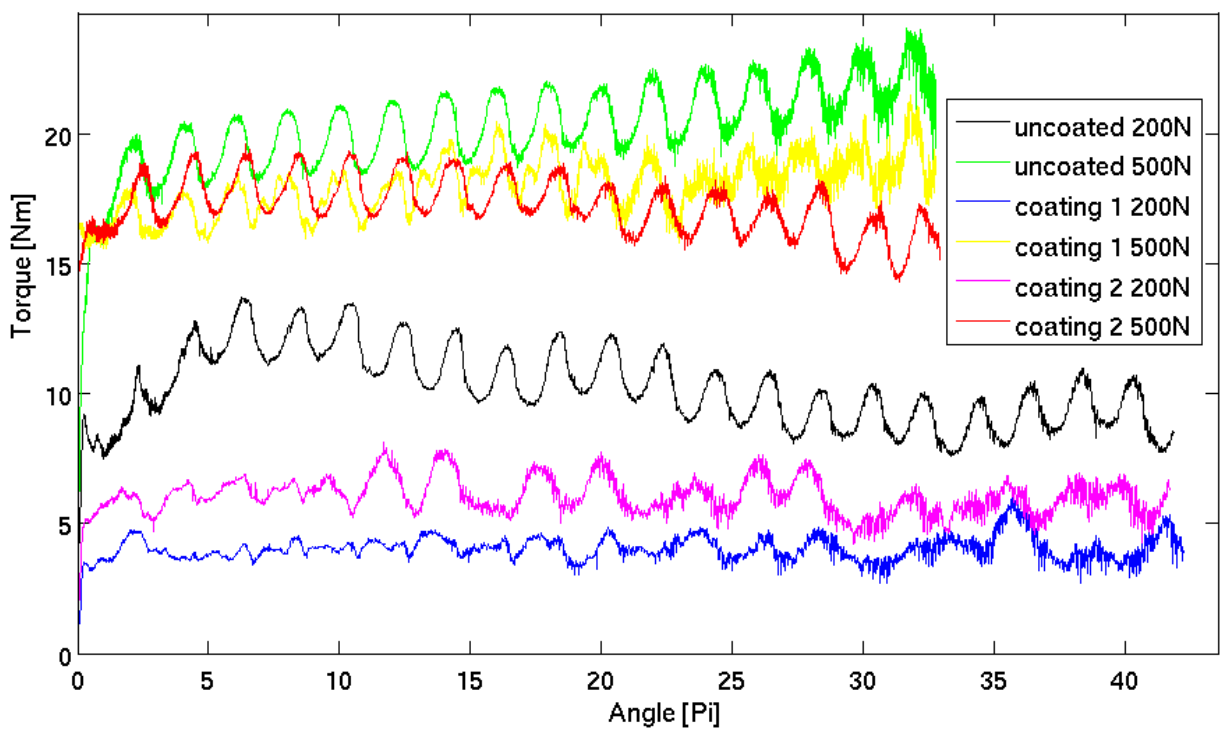

Fig. 7: Measurements for the tested cases.

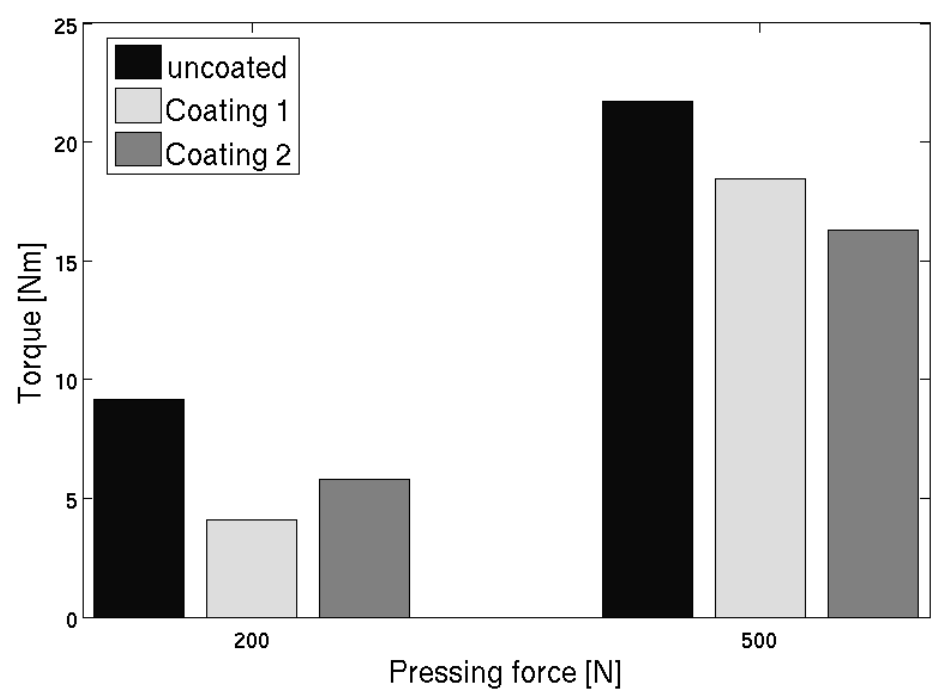

Fig. 8: Dependency of friction on temperature and axial force.

\section{Conclusions}

The new cone-friction test enables the control of temperature, pressure and relative velocity, yielding the measurement of friction dependent on these process parameters. Compared to conventional friction test setups, conditions similar to extrusion are better represented. This makes the information obtained from a cone-friction test valued especially for design of extrusion tools. The presented experimental results show the applicability of this new test for further investigations. As a complementary study, the results of these and further tests will be used in extrusion simulations for which the comparison with the reality is available.

\section{References}

[1] P. Hora, C. Karadogan, L. Tong: Numerische Modellierung thermischer und tribologischer Randbedingungen, Conference Proceedings: Extrusion Zurich (2005)

[2] C. Karadogan, F. Vanini, L. Tong, P. Hora: State of the Art and Potential Development of Digital Extrusion Modeling, Light Metal Age, Volume 63 (May 2005), No. 3, pp. 40-43. 
[3] C. Karadogan; L. Tong, P. Hora: An Improved Modeling of Friction for Extrusion Simulations, 10TH ESAFORM CONFERENCE ON MATERIAL FORMING. AIP Conference Proceedings, Volume 907 (2007), pp. 1325-1330.

[4] B. Post-Pedersen; T. Wanheim, N. Bay, Verification of a friction test for warm and hot forging, ICFT '98, (1998), Issue: 3 pp. 253-261

[5] O. Cora, M. Akkok, H. Darendeliler, Modelling of variable friction in cold forging, Proceedings of the institution of Mechanical Engineers Part J-Journal of Engineering Tribology (2008), Volume 222 Issue: J7 Pages: 899-908

[6] K. Lange, Handbook of metal forming, McGraw-Hill Int. Book Company, USA (1985)

[7] Joachim Maier, CVD Coated Aluminium Extrusion Dies, Conference Proceedings: Extrusion Workshop (2009), Dortmund 